\title{
Pharmacological enhancement of fibrinolytic activity of blood ${ }^{1}$
}

\author{
G. R. FEARNLEY AND R. CHAKRABARTI
}

From the Gloucestershire Royal Hospital, Gloucester

The studies to be described depend on the hypothesis that the spontaneous fibrinolytic activity of normal blood, demonstable in vitro, reflects an active fibrinolytic mechanism in vivo whose function is to ensure the removal of deposited fibrin. This involves two assumptions, first that a somewhat artificial test in vitro is evidence of activity in vivo, and second that fibrin is being commonly deposited on the walls of blood vessels. The first assumption receives support from the predictable response of fibrinolytic activity of blood to well-established methods of influencing it, namely, physical exercise and injection of adrenaline, and the second becomes tenable when the discrepancy between atherosclerosis and vascular occlusion is considered. A visit to the post-mortem room reveals that atherosclerosis can be widespread and longstanding, whereas occlusion of affected vessels is limited to time and place. There must exist effective mechanisms to counteract the tendency to thrombosis, of which at present little is known; these may well be multifactorial, but on grounds of probability alone fibrinolysis merits scrutiny in this respect.

The activity demonstrable in vitro is due to an activator of plasminogen (Flute, 1959) and since this activator is strongly absorbed by fibrin (Fearnley, 1953), it is not difficult to conceive of its functioning as a fibrin-clearing mechanism, without impairing haemostasis (Fearnley and Ferguson, 1958; Fearnley, 1961). If this hypothesis is entertained, the problem of vascular occlusion can be visualized as a tripod whose limbs are atherosclerosis, coagulation, and fibrinolysis. The past 20 years have seen attempts to diminish the coagulability of the blood, again on the basis of change in a somwehat artificial test-tube measurement, and while there is general agreement about the value of anticoagulant therapy in the treatment and prevention of venous thrombosis and embolism, it is becoming apparent that its benefits in arterial disease, whether for the treatment of acute episodes

${ }^{1}$ These studies were supported by a grant from the South Western Regional Hospital Board. or when used prophylactically, are of a margina order.

Thrombolytic agents like streptokinase, and $\dot{\omega}_{\omega}^{\omega}$ more recently, urokinase, have been subjected to nothing like the trial enjoyed by anticoagulants, but to the clinician they seem to have an uncertain future except perhaps in situations like arterial thrombosisz of a limb where time is on the therapist's side Certainly they cannot in their present form be usedprophylactically.

Some $60 \%$ of 80 patients with occlusive vascular disease have been found by us to have 'low' bloof fibrinolytic activity, as judged by dilute blood-clos lysis times repeatedly greater than seven hours when studied in the fasting, resting state. Whethero or not this reflects what might be found in th® population as a whole, it does mean that bloof fibrinolytic activity is lower in some people than in others, including a sizeable proportion of patient? with ischaemic disease; and this contrasts with. coagulability, as measured by the prothrombin time which does not differ significantly among arterio pathic patients.

For these reasons during the past five years we have been attempting to find a drug which, given bo mouth, will increase spontaneous fibrinolytic activity in those in whom it appears to be low. The object is prophylactic, and the validity of the hypothesis on which it is based can be tested only b. clinical trial. The first problem, however, is to fing such a drug. A clue that it might be possible to influence the spontaneous activity of blood pharmas cologically came from the observation that when afo injection of insulin is given to diabetics a biphasie fibrinolytic response ensues, consisting of ate initial reduction followed by a rebound increase of fibrinolytic activity (Fearnley, Vincent. an $\widetilde{\Phi}^{+}$ Chakrabarti, 1959).

Before reviewing the effect of various druge investigated, the procedure will be described.

Because of the numerous factors which influenco the lysis times of individuals, it has been foun essential to limit observations to in-patients studied 


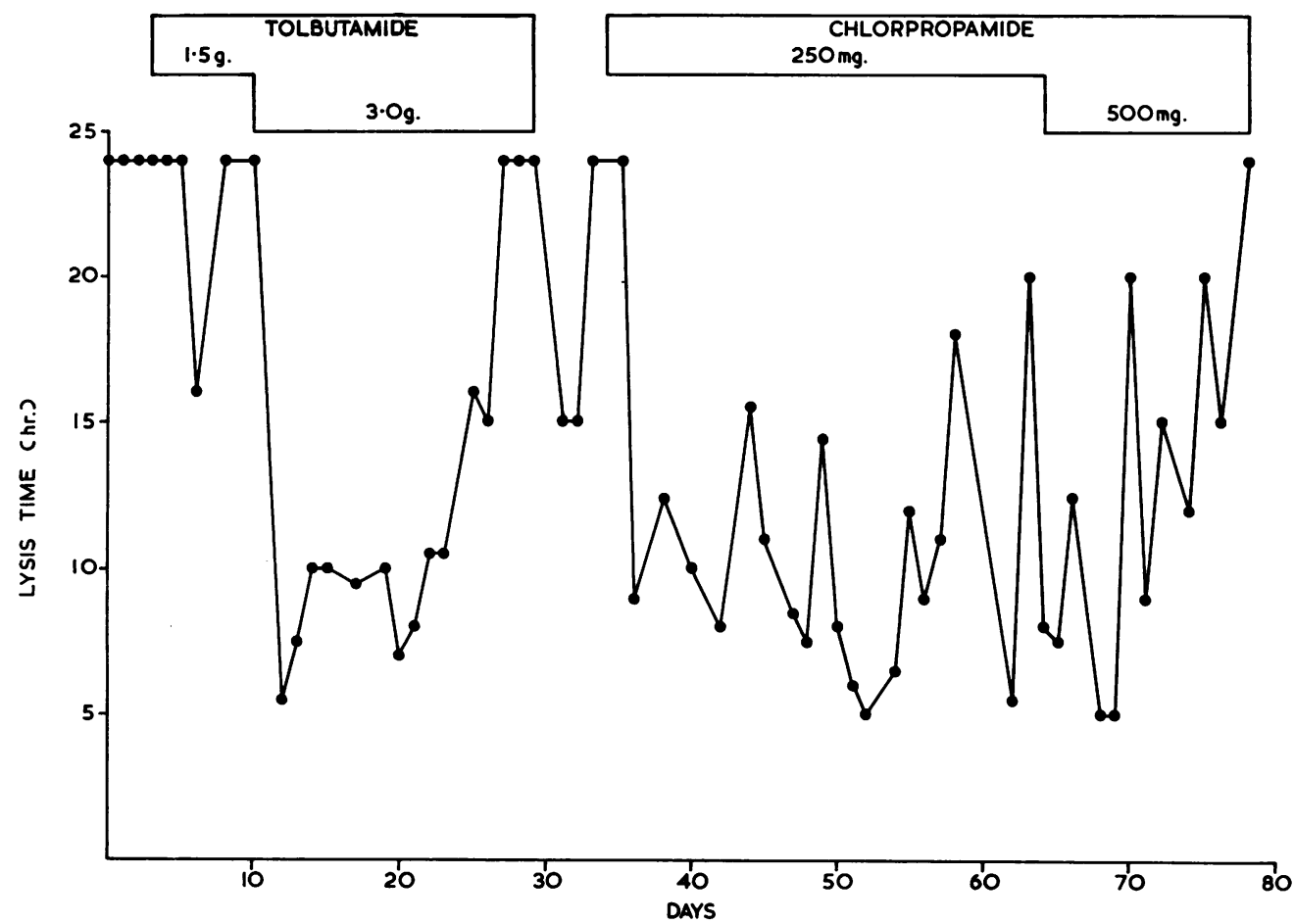

FIG. 1. Temporary reduction of dilute blood-clot lysis time (increase of blood fibrinolytic activity) by tolbutamide and chlorpropamide in a woman with ischaemic heart disease. Both drugs produced a reduction in lysis time, but resistance developed to each drug in spite of increasing dosage.

in the fasting, resting state at 8.30 a.m. Observations of this kind on out-patients are in our experience of little value. Even when in-patients are studied in this way, some show fluctuations of fibrinolytic activity from day to day. Nevertheless if sufficient observations are made, ideally between five and 10 , it is usually possible to categorize an individual's fibrinolytic status at this time of day under these conditions, and thus to obtain a base-line against which can be evaluated the effect of medication. When a drug has been found to influence fibrinolytic activity significantly, in order to determine whether the effect is maintained the patient can be readmitted three months later so that six daily determinations can be made for comparison with those at the time of his discharge from hospital.

\section{SULPHONYL-UREA DRUGS}

The effect of insulin suggested that the sulphonylurea drugs, tolbutamide and chlorpropamide, might increase fibrinolysis in non-diabetic people. Investigation showed that they do (Fearnley, Ferguson, Chakrabarti, and Vincent, 1960), but it later became apparent that resistance to this effect develops after a time in the majority of patients. Figure 1 shows the temporary effect of tolbutamide and chlorpropamide on fibrinolysis in a woman with ischaemic heart disease. Both drugs produced a reduction of the dilute blood-clot lysis time but resistance developed to each in spite of increasing dosage.

\section{MALE HORMONES}

Because of a sex difference in blood fibrinolytic activity noted during an investigation of fibrinolysis in diabetes mellitus (Fearnley, Chakrabarti, and Avis, 1963), the effect of male sex hormones was studied. Testosterone and nandrolone were each found to increase blood fibrinolytic activity when given by frequent injection in large dosage (Fearnley and Chakrabarti, 1962). The fibrinolytic effect appeared to be sustained, but the frequent injections were a disadvantage.

Anabolic steroids given by mouth were then investigated, but the same disappointing development of resistance found with the sulphonyl ureas was encountered. Figure 2 shows the temporary 


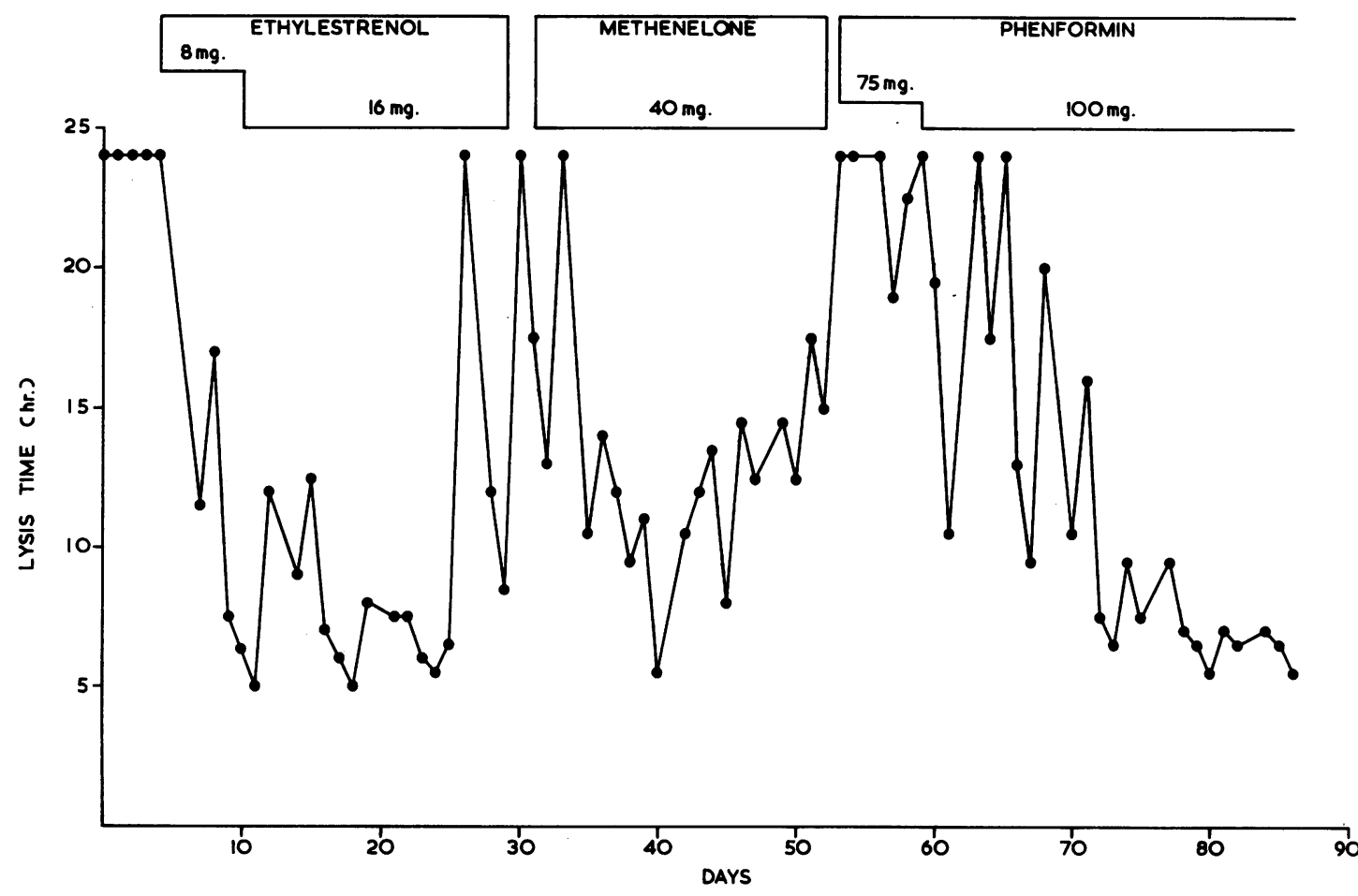

FIG. 2. Temporary reduction of lysis time by ethylestrenol and by methenelone in a man with ischaemic limb disease. Phenformin gave a reduction of lysis time, which was found to be sustained when the patient was re-admitted three months later.

fibrinolytic effect of ethylestrenol and methenelone with resistance developing to each in a man with ischaemic limb disease and very low fibrinolytic activity. He subsequently responded to phenformin, and the response was found to be maintained when he was re-admitted for assessment three months later (Table I, case 1).

Clofibrate (Atromid) was given to two patients both of whom showed a temporary fibrinolytic response, followed by resistance (Fearnley, 1963).

TABLE I

EFFECT OF PHENFORMIN ON FIBRINOLYSIS IN ATHEROSCLEROSIS

\begin{tabular}{lll} 
No. & Sex & Age $(y r)$. \\
& & \\
\hline 1 & M. & 68 \\
2 & M. & 74 \\
3 & M. & 42 \\
4 & M. & 58 \\
5 & F. & 67 \\
6 & M. & 72 \\
7 & M. & 57 \\
8 & M. & 64 \\
9 & M. & 48
\end{tabular}

Diagnosis

Mean Lysis Time (Hours) ${ }^{1}$

\begin{tabular}{|c|c|c|c|}
\hline & Mean $L_{j}$ & $(\text { Hours })^{1}$ & \\
\hline & Control & On Discharge & At Three Months \\
\hline & $\begin{array}{c}24+ \\
24+ \\
13 \cdot 3 \\
13 \cdot 2 \\
12 \cdot 3 \\
9 \cdot 0 \\
9 \cdot 3 \\
6.5 \\
6.0\end{array}$ & $\begin{array}{l}6.5 \\
5.5 \\
6.1 \\
9.8 \\
4.7 \\
6.2 \\
4.0 \\
4.4\end{array}$ & $\begin{array}{l}6 \cdot 8 \\
6 \cdot 0 \\
5 \cdot 1 \\
6 \cdot 7 \\
7 \cdot 5 \\
5 \cdot 2 \\
6 \cdot 6 \\
4 \cdot 9 \\
4 \cdot 8\end{array}$ \\
\hline Mean & $13 \cdot 1$ & 5.9 & 6.0 \\
\hline & $\begin{array}{r}12 \cdot 4 \\
14 \cdot 2 \\
7 \cdot 9 \\
5 \cdot 5 \\
4 \cdot 8 \\
9 \cdot 8 \\
12 \cdot 3\end{array}$ & $\begin{array}{r}6 \cdot 5 \\
7 \cdot 8 \\
4 \cdot 7 \\
4 \cdot 3 \\
3 \cdot 7 \\
9 \cdot 0 \\
24+\end{array}$ & $\begin{array}{l}- \\
z \\
- \\
-\end{array}$ \\
\hline
\end{tabular}

Ischaemia of leg

Ischaemia of leg

Ischaemia of leg

Ischaemic heart disease

Ischaemic heart disease

Ischaemic heart disease

Ischaemic heart disease

Ischaemia of leg

Ischaemic heart disease

$\begin{array}{ll}\text { M. } & \mathbf{6 8} \\ \text { F. } & \mathbf{7 4} \\ \text { F. } & \mathbf{6 8} \\ \text { M. } & 49 \\ \text { M. } & 66 \\ \text { M. } & \mathbf{5 8} \\ \text { M. } & \mathbf{6 2}\end{array}$


PHENFORMIN

The effect of the sulphonyl ureas suggested a trial of phenformin, and this drug has been found to increase blood fibrinolytic activity without apparently inducing resistance to its effect. In Table I are shown the results of treating 16 patients with ischaemic conditions. The control lysis time is the mean of five daily determinations following the patient's admission to hospital; the lysis time on discharge is the mean of four daily determinations after the patient had been receiving phenformin for three to four weeks; in those patients who were re-admitted for assessment three months after starting the drug the mean lysis time during a re-admission is derived from four to six daily determinations. Phenformin gave a reduction of lysis time in 14 of the patients (nos. 1-14). An insignificant change occurred in patient no. 15, and patient no. 16 was completely resistant, the lysis time at the end of admission being in fact longer than during the control period. Of the nine patients re-admitted for further study after they had been receiving phenformin for three months, all showed a sustained increase of fibrinolytic activity.

At first the drug was given as tablets, $25 \mathrm{mg}$. thrice daily, increasing to four times daily after a week; latterly as long-acting capsules, $50 \mathrm{mg}$. twice daily, increasing after a week in some patients to $150 \mathrm{mg}$. daily. In common with some of the other drugs found to increase fibrinolytic activity, phenformin takes seven to 21 days to achieve a sustained reduction of lysis time. The time required seems to vary with the individual rather than with the initial lysis time, and the sustained effect tends to be preceded by a period during which the lysis time fluctuates, suggesting that the stimulus to fibrinolytic activity is being opposed by some homeostatic counter-mechanism.

Phenformin has the advantage of not lowering blood glucose levels in non-diabetic people, and appears to be safe. It causes gastric intolerance, however, with increasing dosage, and this puts a limitation on dose and hence on effectiveness. We have found that while most men will tolerate $150 \mathrm{mg}$. of the drug daily in the form of long-acting capsules, few women can take more than $100 \mathrm{mg}$. daily without nausea and vomiting. Undoubtedly in some people the latter dose is insufficient to produce an increase of fibrinolytic activity. Nevertheless phenformin seems to be the most effective drug, excepting corticosteroids, that we have yet found for this purpose.

\section{CORTICOSTEROIDS}

Adrenocorticotrophic hormone (A.C.T.H.), prednisone, and cortisone have been found to produce a marked increase of blood fibrinolytic activity in patients with inflammatory and noninflammatory conditions (Chakrabarti, Fearnley, and Hocking, 1964). This may have a bearing on their anti-inflammatory actions, which will not be elaborated here. However, this is yet another class of drugs, hormonal in type, which increases fibrinolysis. Figure 3 shows the effect of prednisone in an elderly woman with heart block, whose lysis times during the control period lay between 10 and 12 hours. Twenty-four hours after starting prednisone, $20 \mathrm{mg}$. daily, the lysis time fell to four hours and thereafter to two and a half hours to three. Reduction of dosage from $20 \mathrm{mg}$. to $15 \mathrm{mg}$. daily was followed by prolongation of the lysis time to six to eight hours for eight days, after which it settled at a level

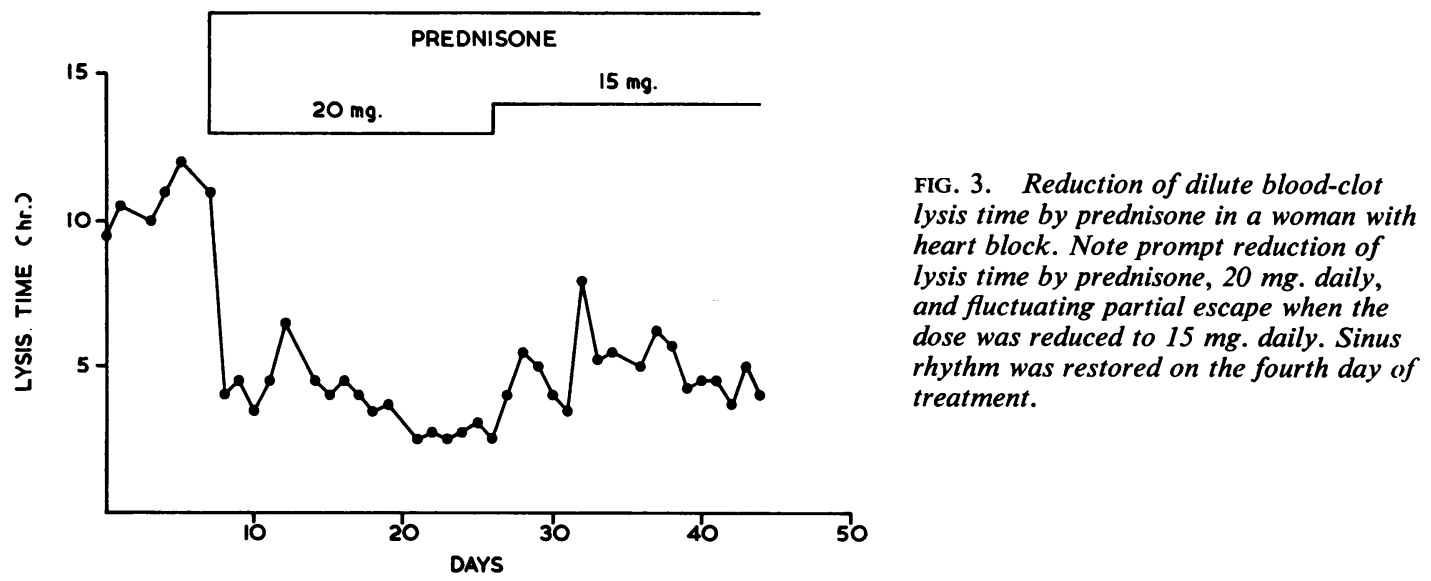




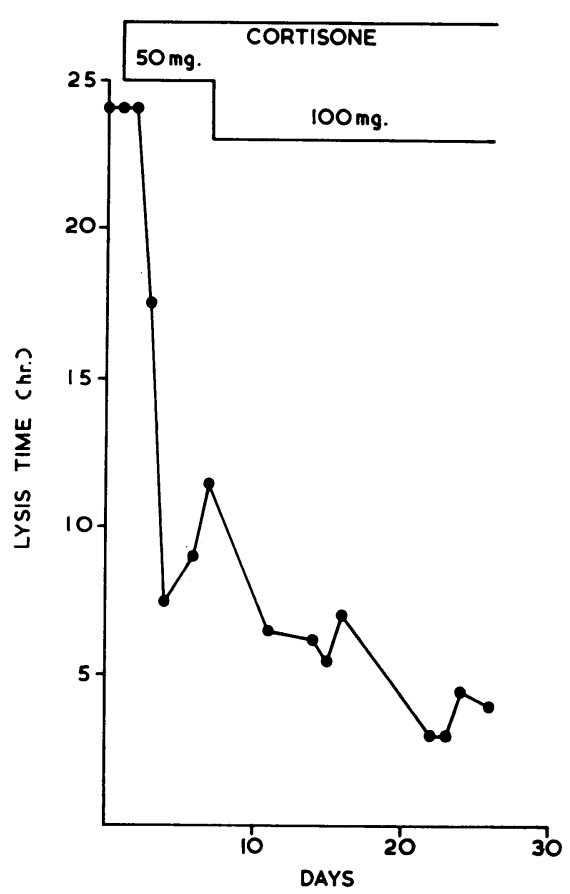

FIG. 4. Reduction of lysis time by cortisone in a man with hitherto untreated Addison's disease.

of four to five hours. Sinus rhythm was restored on the fourth day of treatment.

Figure 4 illustrates the reduction of lysis time by cortisone in a man with hitherto untreated Addison's disease, an example of the effect of replacement therapy on fibrinolysis in a patient with adrenocortical insufficiency.

\section{CONCLUSIONS}

The multiplicity of drugs which appear capable of increasing the spontaneous fibrinolytic activity of blood, some having a temporary and some a sustained effect, suggests that others exist or may be synthesized free from the disadvantages of those described. Phenformin seems to be the most satisfactory drug we have yet studied, but gastric intolerance limits dosage and hence effectiveness in some people.

Clinical impressions in this field are notoriously unreliable, but we have seen healing of early gangrene in some of the patients treated with large doses of testosterone and also in some treated with phen- $\overrightarrow{\overrightarrow{0}}$ formin. This is a short-term effect whose validityo could be tested by the study of a large number of $\frac{}{0}$ patients. The long-term effects of increasing blood $\overline{\frac{\bar{m}}{7}}$ fibrinolytic activity in arteriopathic patients remain $\frac{\widehat{\Phi}}{2}$ to be evaluated, but $a$ priori this would seem to be a therapeutic endeavour no less rational than anticoagulation.

\section{REFERENCES}

Chakrabarti, R., Fearnley, G. R., and Hocking, E. D. (1964). Brit. med. J., 1, 534.

Fearnley, G. R. (1953). Nature (Lond.), 172, 544.

(1961). Lancet, 1, 992.

(1963). Ibid., 2, 148.

—, and Chakrabarti, R. (1962). Ibid., 2, 128.

,-- , and Avis, P. R. D. (1963). Brit. med. J., $1,921$.

- and Ferguson, J. (1958). Clin. Sci., 17, 555.

- Chakrabarti, R., and Vincent, C. T. (1960). Lancet, 2, 622 윽

-, Vincent, C. T., and Chakrabarti, R. (1959). Lancet, 2, 1067.

Flute, P. T. (1959). Proc. 7th Congr. europ. Soc. Haematol., part II p. 894.

DR. MACFARLANE asked whether any of the drugs used by Dr. Fearnley to increase fibrinolytic activity was associ-: ated with flushing or change of blood pressure, in view of the fact that exercise, emotion, adrenaline, and nicotinic acid all created some circulatory disturbance.

DR. FEARNLEY replied that none of the substances he had used produced any measurable change of this kind.

DR. EMERY asked for information about the effect of exercise on fibrinolysis.

DR. FEARNLEY replied that Dr. MacFarlane had been the first person to demonstrate the effect of exercise. He, Dr. Fearnley, wondered whether it had something to do with the increased blood flow, and hence increased venous return of the exercised limbs. He mentioned work done in America and shortly to be published, $\frac{\bar{\gamma}}{3}$ showing that exercise of one limb will produce increased. fibrinolytic activity in the venous blood flow of theo exercised limb compared with the opposite unexercised limb.

DR. MACFARLANE asked Dr. Fearnley his views about the Kwaan hypothesis of constriction of the vasa vasorumo in relation to fibrinolytic activity.

DR. FEARNLEY felt that the thesis in general might well be true, but that the data were somewhat questionable because venous occlusion, which Kwaan et al. claimed to have no effect on fibrinolytic activity, played such aw prominent part in their experiments. Unfortunately ito had been clearly shown that venous occlusion was one of the best ways of raising fibrinolytic activity. 